\title{
STATIONARITY AND REGULARITY CONCEPTS FOR SET SYSTEMS
}

\author{
A. Kruger ${ }^{1}$ \\ ${ }^{1}$ University of Ballarat, School of Information Technology and Mathematical Sciences, Centre \\ of Information and Applied Optimization, Ballarat, Australia, a.kruger@ballarat.edu.au
}

\begin{abstract}
The paper investigates stationarity and regularity concepts for set systems in a normed space. Several primal and dual constants characterizing these properties are introduced and the relations between the constants are established. The equivalence between the regularity property and the strong metric inequality is established. The extended extremal principle is formulated.
\end{abstract}

keywords: nonsmooth analysis, normal cone, optimality, extremality, stationarity, regularity, set-valued mapping, Asplund space

\section{Introduction}

Starting with the pioneering work by Dubovitskii and Milyutin [2] it is quite natural when dealing with optimality conditions to reformulate optimality in the original optimization problem as a (some kind of) extremal behaviour of a certain system of sets. An easy example is a problem of unconditional minimization of a real-valued function $\varphi: X \rightarrow R$. If $x^{\circ} \in X$ one can consider the sets $\Omega_{1}=\operatorname{epi} \varphi=\{(x, \mu) \in X \times R: \varphi(x) \leq \mu\}$ (the epigraph of $\varphi$ ) and $\Omega_{2}=X \times\left\{\mu: \mu \leq \varphi\left(x^{\circ}\right)\right\}$ (the lower halfspace). The local optimality of $x^{\circ}$ is then equivalent to the condition $\Omega_{1} \cap$ int $\Omega_{2} \cap B_{\rho}\left(x^{\circ}\right)=\emptyset$ for some $\rho>0$.

Besides extremality, stationarity and regularity concepts for set systems can be defined in a natural way. Regularity properties of set systems are closely related to similar properties of multifunctions. They can play the role of constraint qualifications in optimization problems.

The paper is organized as follows. Several primal constants characterizing the mutual arrangement of sets in a normed space are introduced in Section 2. Based on these constants the extremality, stationarity and regularity properties for the set system are defined. Two special cases are considered in Section 3: a system of convex sets and a system of (not necessarily convex) cones. In Section 4 two more primal constants based on comparing point-to-set distances are introduced. They give rise to another two regularity properties: the metric

Please use the following format when citing this chapter:

Kruger, A., 2006, in IFIP International Federation for Information Processing, Volume 202, Systems, Control, Modeling and Optimization, eds. Ceragioli, F., Dontchev, A., Furuta, H., Marti, K., Pandolfi, L., (Boston: Springer), pp. 205-214. 
inequality and the strong metric inequality. The latter one appears to be equivalent to the regularity property defined in Section 2 . Section 5 is devoted to the dual constants and dual criteria of stationarity and regularity. The extended extremal principle is formulated.

Mainly standard notations are used throughout the paper. The ball of radios $\rho$ centered at $x$ in a normed space is denoted $B_{\rho}(x)$. We write $B_{\rho}$ if $x=0$, and simply $B$ if $x=0$ and $\rho=1$. If $\Omega$ is a set then int $\Omega$ denotes its interior.

\section{Definitions}

Let us consider a system of closed sets $\Omega_{1}, \Omega_{2}, \ldots, \Omega_{n}(n>1)$ in a normed space $X$ with $x^{\circ} \in \cap_{i=1}^{n} \Omega_{i}$.

The following constant can be used for characterizing the mutual arrangement of sets $\Omega_{1}, \Omega_{2}, \ldots, \Omega_{n}$ near $x^{\circ}([9,10])$ :

$$
\begin{aligned}
\theta_{\rho}\left[\Omega_{1}, \ldots, \Omega_{n}\right]\left(x^{\circ}\right)= & \sup \{r \geq 0: \\
& \left.\left(\bigcap_{i=1}^{n}\left(\Omega_{i}-a_{i}\right)\right) \bigcap B_{\rho}\left(x^{\circ}\right) \neq \emptyset, \forall a_{i} \in B_{r}\right\} .
\end{aligned}
$$

It shows how far the sets can be "pushed apart" while still intersecting in a neighborhood of $x^{\circ}$. Evidently $\theta_{\rho}\left[\Omega_{1}, \ldots, \Omega_{n}\right]\left(x^{\circ}\right)$ is nonnegative (and can be equal to $+\infty)$ and nondecreasing as a function of $\rho$.

A slightly more general form of $(0)$ can be of interest ([11]):

$$
\begin{aligned}
\theta_{\rho}\left[\Omega_{1}, \ldots, \Omega_{n}\right]\left(\omega_{1}, \ldots, \omega_{n}\right)=\sup \{r \geq 0: \\
\left.\left(\bigcap_{i=1}^{n}\left(\Omega_{i}-\omega_{i}-a_{i}\right)\right) \bigcap B_{\rho} \neq \emptyset, \forall a_{i} \in B_{r}\right\} .
\end{aligned}
$$

This constant corresponds to the case when instead of the common point $x^{\circ} \in \cap_{i=1}^{n} \Omega_{i}$ each of the sets $\Omega_{i}$ is considered near its own point $\omega_{i} \in \Omega_{i}$, $i=1,2, \ldots, n$. The sets do not need to be intersecting. It is equivalent to considering the system of translated sets $\Omega_{1}-\omega_{1}, \Omega_{2}-\omega_{2}, \ldots, \Omega_{n}-\omega_{n}$ near 0:

$$
\theta_{\rho}\left[\Omega_{1}, \ldots, \Omega_{n}\right]\left(\omega_{1}, \ldots, \omega_{n}\right)=\theta_{\rho}\left[\Omega_{1}-\omega_{1}, \ldots, \Omega_{n}-\omega_{n}\right](0) .
$$

If $\omega_{1}=\omega_{2}=\ldots=\omega_{n}=x^{\circ}$ then, of course,

$$
\theta_{\rho}\left[\Omega_{1}, \ldots, \Omega_{n}\right]\left(\omega_{1}, \ldots, \omega_{n}\right)=\theta_{\rho}\left[\Omega_{1}, \ldots, \Omega_{n}\right]\left(x^{\circ}\right) .
$$

If $(0)$ or $(1)$ is positive more precise estimates of regularity/stationarity can be obtained based on using the "linearized" constants:

$$
\begin{aligned}
& \theta\left[\Omega_{1}, \ldots, \Omega_{n}\right]\left(x^{\circ}\right)=\liminf _{\rho \rightarrow+0} \theta_{\rho}\left[\Omega_{1}, \ldots, \Omega_{n}\right]\left(x^{\circ}\right) / \rho, \\
& \theta\left[\Omega_{1}, \ldots, \Omega_{n}\right]\left(\omega_{1}, \ldots, \omega_{n}\right)= \\
& =\liminf _{\rho \rightarrow+0} \theta_{\rho}\left[\Omega_{1}, \ldots, \Omega_{n}\right]\left(\omega_{1}, \ldots, \omega_{n}\right) / \rho .
\end{aligned}
$$


Finally, one can define one more limiting constant based on (4):

$$
\hat{\theta}\left[\Omega_{1}, \ldots, \Omega_{n}\right]\left(x^{\circ}\right)=\liminf _{\substack{\Omega_{i} \\ \omega_{i} \rightarrow x^{\circ}}} \theta\left[\Omega_{1}, \ldots, \Omega_{n}\right]\left(\omega_{1}, \ldots, \omega_{n}\right) .
$$

The notation $\omega \stackrel{\Omega}{\rightarrow} x$ in (5) means that $\omega \rightarrow x$ with $\omega \in \Omega$.

The constants (3)-(5) are in a sense derivative-like objects. (3) and (4) can be considered as analogs of the usual derivative, while (5) has some properties of the strict derivative: it accumulates information about local properties of the sets not only at a given point but also at all nearby points.

All the constants (0)-(5) are nonnegative. When investigating extremalitystationarity-regularity properties of the set system one needs to check whether the corresponding constant is zero or strictly positive.

DEFINITION 1 The system of sets $\Omega_{1}, \Omega_{2}, \ldots, \Omega_{n}$ is

(i) extremal at $x^{\circ}$ if $\theta_{\rho}\left[\Omega_{1}, \ldots, \Omega_{n}\right]\left(x^{\circ}\right)=0$ for all $\rho>0$.

(ii) locally extremal at $x^{\circ}$ if $\theta_{\rho}\left[\Omega_{1}, \ldots, \Omega_{n}\right]\left(x^{\circ}\right)=0$ for some $\rho>0$.

(iii) stationary at $x^{\circ}$ if $\theta\left[\Omega_{1}, \ldots, \Omega_{n}\right]\left(x^{\circ}\right)=0$.

(iv) weakly stationary at $x^{\circ}$ if $\hat{\theta}\left[\Omega_{1}, \ldots, \Omega_{n}\right]\left(x^{\circ}\right)=0$.

(v) regular at $x^{\circ}$ if $\hat{\theta}\left[\Omega_{1}, \ldots, \Omega_{n}\right]\left(x^{\circ}\right)>0$.

Proposition $2(i) \Rightarrow$ (ii) $\Rightarrow$ (iii) $\Rightarrow$ (iv) in Definition 1 .

Opposite implications are not true in general.

The notion of (local) extremality of the set system was introduced (in a different but equivalent way) in [12], where dual necessary conditions were formulated. This result currently known as the extremal principle has had numerous applications to different optimization problems (see [13]).

Conditions (iii) and (IV) give natural extensions of the notion of local extremality. Condition (iii) corresponds to the traditional concept of stationarity in optimization theory, while (iv) means that arbitrarily close to $x^{\circ}$ there exist points whose properties are arbitrarily close to the traditional stationarity property. The first version of the weak stationarity property was defined (under a different name) in [6] (see also [7, 8]).

Stationarity and regularity properties of set systems were considered in [10, $11]$.

Regularity of the set system is a natural counterpart of the weak stationarity property. It is closely related to the metric regularity of multifunctions $[4,5]$ and can be used e.g. when formulating constraint qualifications in mathematical programming. 
The condition $\theta\left[\Omega_{1}, \ldots, \Omega_{n}\right]\left(x^{\circ}\right)>0$ also defines a kind of regularity which is weaker than the one defined in part (iv) of Definition 1. It can be referred to as weak regularity. We will not use this concept in the current paper.

The next proposition gives an equivalent definition of regularity.

Proposition 3 The system of sets $\Omega_{1}, \Omega_{2}, \ldots, \Omega_{n}$ is regular at $x^{\circ}$ if and only if there exists an $\alpha>0$ and $a \delta>0$ such that

$$
\left(\bigcap_{i=1}^{n}\left(\Omega_{i}-\omega_{i}-a_{i}\right)\right) \bigcap B_{\rho} \neq \emptyset
$$

for all $\rho \in(0, \delta], \omega_{i} \in \Omega_{i} \cap B_{\delta}\left(x^{\circ}\right), a_{i} \in B_{\alpha \rho}, i=1,2, \ldots, n$.

$\hat{\theta}\left[\Omega_{1}, \ldots, \Omega_{n}\right]\left(x^{\circ}\right)$ equals to the exact upper bound of all such $\alpha$.

\section{Stationarity and regularity of convex set and cone systems}

In the convex case, as one could expect, the concepts of extremality and local extremality coincide and appear to be equivalent to both stationarity and weak stationarity.

Proposition 4 (See [10]) Let $\Omega_{1}, \Omega_{2}, \ldots, \Omega_{n}$ be convex.

(i) If $\theta_{\rho}\left[\Omega_{1}, \ldots, \Omega_{n}\right]\left(x^{\circ}\right)>0$ for some $\rho>0$ then $\theta_{\rho}\left[\Omega_{1}, \ldots, \Omega_{n}\right]\left(x^{\circ}\right)>0$ for all $\rho>0$.

(ii) The function $\rho \rightarrow \theta_{\rho}\left[\Omega_{1}, \ldots, \Omega_{n}\right]\left(x^{\circ}\right) / \rho$, considered on the set of positive numbers, is nonincreasing.

(iii) $\theta\left[\Omega_{1}, \ldots, \Omega_{n}\right]\left(x^{\circ}\right)=\sup _{\rho>0} \theta_{\rho}\left[\Omega_{1}, \ldots, \Omega_{n}\right]\left(x^{\circ}\right) / \rho$.

(iv) $\hat{\theta}\left[\Omega_{1}, \ldots, \Omega_{n}\right]\left(x^{\circ}\right)=\theta\left[\Omega_{1}, \ldots, \Omega_{n}\right]\left(x^{\circ}\right)$.

(v) $($ i $) \Leftrightarrow$ (ii) $\Leftrightarrow$ (iii) $\Leftrightarrow$ (iv) in Definition 1 .

(vi) If int $\Omega_{i} \neq \emptyset, i=1,2, \ldots, n-1$, then the first four conditions in Definition 1 are equivalent to

$$
\bigcap_{i=1}^{n-1} \operatorname{int} \Omega_{i} \bigcap \Omega_{n}=\emptyset
$$

while condition $(v)$ is equivalent to

$$
\bigcap_{i=1}^{n-1} \operatorname{int} \Omega_{i} \bigcap \Omega_{n} \neq \emptyset .
$$


As it follows from part (v) of Proposition 4, under the assumption that all but one sets have nonempty interior, all defined above extremality and stationarity notions reduce in the convex case to the traditional condition (7). Note that the initial definitions make sense for convex sets even without the assumption that the sets have nonempty interior.

Proposition 5 (See [10]) Let $\Omega_{1}, \Omega_{2}, \ldots, \Omega_{n}$ be cones .

(i) If $\omega_{i} \in \Omega_{i}, i=1,2, \ldots, n$, and $\rho>0$ then

$$
\theta_{\rho}\left[\Omega_{1}, \ldots, \Omega_{n}\right]\left(\omega_{1}, \ldots, \omega_{n}\right)=\rho \theta_{1}\left[\Omega_{1}, \ldots, \Omega_{n}\right]\left(\omega_{1} / \rho, \ldots, \omega_{n} / \rho\right) .
$$

In particular, $\rho \rightarrow \theta_{\rho}\left[\Omega_{1}, \ldots, \Omega_{n}\right](0)$ is positively homogeneous:

$$
\theta_{\rho}\left[\Omega_{1}, \ldots, \Omega_{n}\right](0)=\rho \theta_{1}\left[\Omega_{1}, \ldots, \Omega_{n}\right](0) .
$$

(ii) If $\omega_{i} \in \Omega_{i}, i=1,2, \ldots, n$, then

$$
\theta\left[\Omega_{1}, \ldots, \Omega_{n}\right]\left(\omega_{1}, \ldots, \omega_{n}\right)=\liminf _{t \rightarrow \infty} \theta_{1}\left[\Omega_{1}, \ldots, \Omega_{n}\right]\left(t \omega_{1}, \ldots, t \omega_{n}\right) .
$$

In particular, $\theta\left[\Omega_{1}, \ldots, \Omega_{n}\right](0)=\theta_{1}\left[\Omega_{1}, \ldots, \Omega_{n}\right](0)$.

(iii) $\hat{\theta}\left[\Omega_{1}, \ldots, \Omega_{n}\right](0)=\inf _{\omega_{i} \in \Omega_{i}} \theta_{1}\left[\Omega_{1}, \ldots, \Omega_{n}\right]\left(\omega_{1}, \ldots, \omega_{n}\right)$.

(iv) If $x^{\circ}=0$ then (i) $\Leftrightarrow$ (ii) $\Leftrightarrow$ (iii) in Definition 1 and these conditions are equivalent to

$$
\theta_{1}\left[\Omega_{1}, \ldots, \Omega_{n}\right](0)=0 .
$$

(v) The system of sets $\Omega_{1}, \Omega_{2}, \ldots, \Omega_{n}$ is weakly stationary at 0 if and only if

$$
\inf _{\omega_{i} \in \Omega_{i}} \theta_{1}\left[\Omega_{1}, \ldots, \Omega_{n}\right]\left(\omega_{1}, \ldots, \omega_{n}\right)=0 .
$$

(vi) The system of sets $\Omega_{1}, \Omega_{2}, \ldots, \Omega_{n}$ is regular at 0 if and only if there exists $\alpha>0$ such that

$$
\theta_{1}\left[\Omega_{1}, \ldots, \Omega_{n}\right]\left(\omega_{1}, \ldots, \omega_{n}\right) \geq \alpha \quad \forall \omega_{i} \in \Omega_{i} .
$$

\section{Metric inequality}

Some other approaches based on comparing distances can be used for characterizing stationarity/regularity properties of set systems. Let $d(\cdot, \cdot)$ be the distance function in $X$ associated with the norm. We will keep the same notation for point-to-set distances. Thus, $d(x, \Omega)=\inf _{\omega \in \Omega}\|x-\omega\|$ is the distance from a point $x$ to a set $\Omega$ and $d(x, \emptyset)=\infty$. The following constant can be useful:

$$
\vartheta\left[\Omega_{1}, \ldots, \Omega_{n}\right]\left(x^{\circ}\right)=\limsup _{x \rightarrow x^{\circ}}\left[d\left(x, \bigcap_{i=1}^{n} \Omega_{i}\right) / \max _{1 \leq i \leq n} d\left(x, \Omega_{i}\right)\right]_{0} .
$$


The "extended" division operation $(\cdot / \cdot)_{\circ}$ is used in $(8)$ to simplify the definition. It makes division by zero legal. The formal rules are as follows:

$1(\alpha / \beta)_{\circ}=\alpha / \beta$, if $\beta \neq 0$

$2(\alpha / 0)_{\circ}=+\infty$, if $\alpha>0$;

$3(\alpha / 0)_{\circ}=-\infty$, if $\alpha<0$;

$4(0 / 0)_{\circ}=0$.

The fourth rule is the most important one here. In the case $x^{\circ} \in$ int $\cap_{i=1}^{n} \Omega_{i}$ it automatically leads to $\vartheta\left[\Omega_{1}, \ldots, \Omega_{n}\right]\left(x^{\circ}\right)=0$. Otherwise, all the points $x \in \cap_{i=1}^{n} \Omega_{i}$ can be ignored when calculating the value of the upper limit in (8).

The "strict" version of (8) looks a little more complicated: small perturbations (shifts) are applied to the sets.

$$
\hat{\vartheta}\left[\Omega_{1}, \ldots, \Omega_{n}\right]\left(x^{\circ}\right)=\limsup _{\substack{x \rightarrow x^{\circ} \\ x_{i} \rightarrow 0}}\left[d\left(x, \bigcap_{i=1}^{n}\left(\Omega_{i}-x_{i}\right)\right) / \max _{1 \leq i \leq n} d\left(x+x_{i}, \Omega_{i}\right)\right]_{\circ} .
$$

When investigating the properties of set systems it can be important to know whether the corresponding constant (8) or (9) is finite.

Proposition 6 The following assertions hold:

(i) $\vartheta\left[\Omega_{1}, \ldots, \Omega_{n}\right]\left(x^{\circ}\right)<\infty$ if and only if there exists $a \beta>0$ and $a \delta>0$ such that

$$
d\left(x, \bigcap_{i=1}^{n} \Omega_{i}\right) \leq \beta \max _{1 \leq i \leq n} d\left(x, \Omega_{i}\right)
$$

for all $x \in B_{\delta}\left(x^{\circ}\right)$.

$\vartheta\left[\Omega_{1}, \ldots, \Omega_{n}\right]\left(x^{\circ}\right)$ coincides with the exact lower bound of all such $\beta$.

(ii) $\hat{\vartheta}\left[\Omega_{1}, \ldots, \Omega_{n}\right]\left(x^{\circ}\right)<\infty$ if and only if there exists $a \beta>0$ and $a \delta>0$ such that

$$
d\left(x, \bigcap_{i=1}^{n}\left(\Omega_{i}-x_{i}\right)\right) \leq \beta \max _{1 \leq i \leq n} d\left(x+x_{i}, \Omega_{i}\right)
$$

for all $x \in B_{\delta}\left(x^{\circ}\right), x_{i} \in B_{\delta}, i=1,2, \ldots, n$.

$\hat{\vartheta}\left[\Omega_{1}, \ldots, \Omega_{n}\right]\left(x^{\circ}\right)$ coincides with the exact lower bound of all such $\beta$.

The condition formulated in part (i) of Proposition 6 is equivalent to the regularity condition known as the metric inequality $[3,4,16]$ (some authors 
consider the sum of distances instead of the maximum in the right-hand side of (10)). The condition in part (ii) can be considered as the strong metric inequality. If $(10)$ is valid for all $x$ then the system of sets is said to be linear regular $[1,15]$. This property is important when investigating convex optimization problems. One can consider some other regularity properties of set systems with interesting relations to linear regularity (see [15]).

(11) is certainly stronger than (10) even in the convex case. Take for instance $\Omega_{1}=\Omega_{2}=\left\{(x, y) \in R^{2}: y=0\right\}$. Then (10) holds true for all $x$ (with $\beta=1$ ) while (11) does not.

The next theorem proved in [10] gives the relation between (9) and (5). It allows to use (9) for characterizing stationarity and regularity properties of set systems.

TheOREM $7 \hat{\vartheta}\left[\Omega_{1}, \ldots, \Omega_{n}\right]\left(x^{\circ}\right)=1 / \hat{\theta}\left[\Omega_{1}, \ldots, \Omega_{n}\right]\left(x^{\circ}\right)$.

COROLLARY 8 The system of sets $\Omega_{1}, \Omega_{2}, \ldots, \Omega_{n}$ is regular at $x^{\circ}$ if and only if $\hat{\vartheta}\left[\Omega_{1}, \ldots, \Omega_{n}\right]\left(x^{\circ}\right)<\infty$.

It follows from Corollary 8 that regularity of a set system implies the metric inequality.

\section{Dual criteria}

The stationarity and regularity properties of set systems were defined above in terms of primal space elements. When the sets are closed these properties admit some dual characterizations in terms of "normal" elements.

Let $X^{*}$ denote the space (topologically) dual to $X$ and $\langle\cdot, \cdot\rangle$ be the bilinear form defining duality between $X$ and $X^{*}$. Recall that the (Fréchet) normal cone to a set $\Omega$ at $x^{\circ} \in \Omega$ is defined as

$$
N\left(x^{\circ} \mid \Omega\right)=\left\{x^{*} \in X^{*}: \limsup _{\substack{\Omega \rightarrow x^{\circ}}} \frac{\left\langle x^{*}, x-x^{\circ}\right\rangle}{\left\|x-x^{\circ}\right\|} \leq 0\right\} .
$$

In the rest of the section the sets $\Omega_{1}, \Omega_{2}, \ldots, \Omega_{n}$ are assumed closed.

Define a "dual" constant:

$$
\begin{aligned}
& \eta\left[\Omega_{1}, \ldots, \Omega_{n}\right]\left(x^{\circ}\right)=\lim _{\delta \rightarrow+0} \inf \left\{\left[\left\|\sum_{i=1}^{n} x_{i}^{*}\right\| / \sum_{i=1}^{n}\left\|x_{i}^{*}\right\|\right]_{\infty}:\right. \\
&\left.x_{i}^{*} \in N\left(x_{i} \mid \Omega_{i}\right), x_{i} \in \Omega_{i} \cap B_{\delta}\left(x^{\circ}\right), i=1, \ldots, n\right\} .
\end{aligned}
$$

Another "extended" division operation $(\cdot, \cdot)_{\infty}$ is used here. It differs from the $(\cdot, \cdot)_{\circ}$ operation, which was used in $(8),(9)$, in the fourth rule definition: 
4. $(0 / 0)_{\infty}=\infty$.

This allows one to exclude the case $x_{1}^{*}=x_{2}^{*}=\cdots=x_{n}^{*}=0$ when calculating the exact lower bound in (12). If this is the only case ( $x^{\circ} \in$ int $\cap_{i=1}^{n} \Omega_{i}$ ) one automatically gets $\eta\left[\Omega_{1}, \ldots, \Omega_{n}\right]\left(x^{\circ}\right)=\infty$.

Using (12) one can define (a kind of) stationarity for the set system $\Omega_{1}, \Omega_{2}, \ldots$, $\ldots, \Omega_{n}$ by the condition $\eta\left[\Omega_{1}, \ldots, \Omega_{n}\right]\left(x^{\circ}\right)=0$ while the inequality $\eta\left[\Omega_{1}, \ldots\right.$ $\left.\ldots, \Omega_{n}\right]\left(x^{\circ}\right)>0$ can be considered as a regularity condition. As it follows from the next proposition the dual stationarity condition can be considered as some generalization of the separation property (for nonconvex set systems).

Proposition 9 (i) $\eta\left[\Omega_{1}, \ldots, \Omega_{n}\right]\left(x^{\circ}\right)=0$ if and only if for any $\delta>0$ there exist elements

$$
\omega_{i} \in \Omega_{i} \cap B_{\delta}\left(x^{\circ}\right), x_{i}^{*} \in N\left(\omega_{i} \mid \Omega_{i}\right), i=1,2, \ldots, n,
$$

such that

$$
\sum_{i=1}^{n}\left\|x_{i}^{*}\right\|=1,\left\|\sum_{i=1}^{n} x_{i}^{*}\right\|<\delta .
$$

(ii) $\eta\left[\Omega_{1}, \ldots, \Omega_{n}\right]\left(x^{\circ}\right)>0$ if and only if there exists $a \gamma>0$ and $a \delta>0$, such that

$$
\left\|\sum_{i=1}^{n} x_{i}^{*}\right\| \geq \gamma \sum_{i=1}^{n}\left\|x_{i}^{*}\right\|
$$

for all $x_{i}^{*} \in N\left(x_{i} \mid \Omega_{i}\right), x_{i} \in \Omega_{i} \cap B_{\delta}\left(x^{\circ}\right), i=1, \ldots, n$.

$\eta\left[\Omega_{1}, \ldots, \Omega_{n}\right]\left(x^{\circ}\right)$ equals to the exact upper bound of all such $\gamma$.

The relation between primal and dual stationarity/regularity conditions is given by the next theorem.

Theorem 10 (SeE [9]) (i) $\hat{\theta}\left[\Omega_{1}, \ldots, \Omega_{n}\right]\left(x^{\circ}\right) \leq \eta\left[\Omega_{1}, \ldots, \Omega_{n}\right]\left(x^{\circ}\right)$.

(ii) If $X$ is Asplund and $\hat{\theta}\left[\Omega_{1}, \ldots, \Omega_{n}\right]\left(x^{\circ}\right)<1$ then

$$
\eta\left[\Omega_{1}, \ldots, \Omega_{n}\right]\left(x^{\circ}\right) \leq \frac{\hat{\theta}\left[\Omega_{1}, \ldots, \Omega_{n}\right]\left(x^{\circ}\right)}{1-\hat{\theta}\left[\Omega_{1}, \ldots, \Omega_{n}\right]\left(x^{\circ}\right)} .
$$

CoROLlaRY $11 \eta\left[\Omega_{1}, \ldots, \Omega_{n}\right]\left(x^{\circ}\right)=0$ then the system of sets $\Omega_{1}, \Omega_{2}, \ldots$, $\Omega_{n}$ is weakly stationary at $x^{\circ}$.

(ii) If $X$ is Asplund then the Extended extremal principle is valid:

The system $\Omega_{1}, \Omega_{2}, \ldots, \Omega_{n}$ is weakly stationary at $x^{\circ}$ if and only if $\eta\left[\Omega_{1}, \ldots\right.$, $\left.\ldots, \Omega_{n}\right]\left(x^{\circ}\right)=0$.

Due to Proposition 2 it follows from the second part of Corollary 11 that in the Asplund space setting the equality $\eta\left[\Omega_{1}, \ldots, \Omega_{n}\right]\left(x^{\circ}\right)=0$ is a necessary 
condition of local extremality of the set system. This result first proved in [12] for spaces admitting an equivalent Fréchet differentiable norm and then extended in [14] to general Asplund spaces, is currently known as the Extremal principle $[13,14]$ and is one of the main tools for deducing necessary optimality conditions in nonsmooth and nonconvex problems.

Taking into account the extremal characterizations of Asplund spaces in [14] one can conclude that asplundity of the space is not only sufficient but also necessary for the Extended extremal principle to be valid. This gives another proof of the well known fact that, being a rather rich subclass of general Banach spaces (see [17]), Asplund spaces provide the appropriate framework for using Fréchet normals and subdifferentials.

THEOREM 12 The following assertions are equivalent:

(i) $X$ is an Asplund space.

(ii) The Extremal principle is valid in $X$.

(iii) The Extended extremal principle is valid in $X$.

\section{References}

[1] H.H. Bauschke, J.M. Borwein, W. Li. Strong conical hull intersection property, bounded linear regularity, Jameson's property $(G)$, and error bounds in convex optimization. Math. Program. 86:135-160, 1999.

[2] A.Y. Dubovitskii, A.A. Milyutin. Extremum problems in the presence of restrictions. U.S.S.R. Comp. Maths. Math. Phys. 5:1-80, 1965.

[3] A.D. Ioffe. Approximate subdifferentials and applications. 3: the metric theory. Mathematika 36:1-38, 1989.

[4] A.D. Ioffe. Metric regularity and subdifferential calculus. Russian Math. Surveys 55:501$558,2000$.

[5] D. Klatte, B. Kummer. Nonsmooth Equations in Optimization: Regularity, Calculus, Methods and Applications. Kluwer Academic Publishers, Dordrecht, 2002.

[6] A.Y. Kruger. On extremality of set systems. Dokl. Nats. Akad. Nauk Belarusi 42:24-28, 1998 (in Russian).

[7] A.Y. Kruger. Strict $(\varepsilon, \delta)$-semidifferentials and extremality conditions. Optimization 51:539-554, 2002.

[8] A.Y. Kruger. On Fréchet subdifferentials. J. Math. Sci. (N. Y.), 116:3325-3358, 2003.

[9] A.Y. Kruger. Weak stationarity: eliminating the gap between necessary and sufficient conditions. Optimization 53:147-164, 2004.

[10] A.Y. Kruger. Stationarity and regularity of set systems. Pacif. J. Optimiz. 1:101-126, 2005.

[11] A.Y. Kruger. About regularity of set systems. Research Report, University of Ballarat, School of Information Technology and Mathematical Sciences, N. 3, 2005.

[12] A.Y. Kruger, B.S. Mordukhovich. Extremal points and the Euler equation in nonsmooth optimization. Dokl. Akad. Nauk BSSR, 24(8);684-687, 1980 (in Russian). 
[13] B.S. Mordukhovich. The extremal principle and its applications to optimization and economics. In Optimization and Related Topics, A. Rubinov and B. Glover, eds., Applied Optimization, Vol. 47, Kluwer Academic Publishers, Dordrecht, 2001, 343-369.

[14] B.S. Mordukhovich, Y. Shao. Extremal characterizations of Asplund spaces. Proc. Amer. Math. Soc. 124:197-205, 1996.

[15] K.F. Ng, W.H. Yang. Regularities and their relations to error bounds. Math. Program., Ser. A, 99:521-538, 2004.

[16] H.V. Ngai, M. Théra. Metric inequality, subdifferential calculus and applications. SetValued Anal. 9:187-216, 2001.

[17] R.R. Phelps. Convex Functions, Monotone Operators and Differentiability, 2nd edition, Lecture Notes in Mathematics, Vol. 1364, Springer-Verlag, New York, 1993. 